\title{
Separable functions of wingless in distal and ventral patterning of the Tribolium leg
}

\author{
Daniela Grossmann • Johannes Scholten • \\ Nikola-Michael Prpic
}

Received: 14 October 2009 /Accepted: 23 November 2009/Published online: 19 December 2009

(C) The Author(s) 2009. This article is published with open access at Springerlink.com

\begin{abstract}
The gene wingless (wg) in Drosophila is an important factor in leg development. During embryonic development $w g$ is involved in the allocation of the limb primordia. During imaginal disk development $w g$ is involved in distal development and it has a separate role in ventral development. The expression pattern of $w g$ is highly conserved in all arthropods (comprising data from insects, myriapods, crustaceans, and chelicerates), suggesting that its function in leg development is also conserved. However, recent work in other insects (e.g. the milkweed bug Oncopeltus fasciatus) argued against a role of $w g$ in leg development. We have studied the role of $w g$ in leg development of the flour beetle Tribolium castaneum. Using stage-specific staggered embryonic RNAi in wildtype and transgenic EGFP expressing enhancer trap lines
\end{abstract}

Communicated by S. Roth

D. Grossmann · N.-M. Prpic $(\bowtie)$

Johann-Friedrich-Blumenbach-Institut für Zoologie und

Anthropologie, Georg-August-Universität Göttingen,

Abteilung Entwicklungsbiologie, GZMB Ernst-Caspari-Haus,

Justus-von-Liebig-Weg 11,

37077 Göttingen, Germany

e-mail: nprpic@uni-goettingen.de

J. Scholten

Department of Biology II,

Ludwig-Maximilians-Universität München,

Großhaderner Straße 2,

82152 Planegg-Martinsried, Germany

Present Address:

J. Scholten

Julius-Schaxel-Institut für Experimentelle Theorie,

Seelheimer Weg 5,

35043 Marburg, Germany we are able to demonstrate separable functions of Tribolium $w g$ in distal and in ventral leg development. The distal role affects all podomeres distal to the coxa, whereas the ventral role is restricted to cells along the ventral midline of the legs. In addition, severe leg defects after injection into early embryonic stages are evidence that $w g$ is also involved in proximal development and limb allocation in Tribolium. Our data suggest that the roles of $w g$ in leg development are highly conserved in the holometabolous insects. Further studies will reveal the degree of conservation in other arthropod groups.

Keywords Wingless · Leg development · Arthropods . Appendage evolution · Tribolium castaneum

\section{Introduction}

The gene wingless $(w g)$ is mainly known for its role in segment polarity specification during body segmentation in Drosophila (reviewed in Sanson 2001). However, wg is also an important factor in Drosophila leg development (e.g., Cohen et al. 1993; Struhl and Basler 1993; Campbell et al. 1993). The leg primordium in Drosophila is already specified in the embryo. At embryonic stage 11 the primordium of the leg is still combined with the wing primordium in the so-called thoracic limb primordium (Cohen et al. 1993). Wg is required for the specification of the entire thoracic limb primordium where it activates the Distal-less (Dll) gene via the early Dll enhancer 304 (Cohen et al. 1993; Kubota et al. 2003; McKay et al. 2009). This early function of $\mathrm{Wg}$ is repressed on the dorsal side by Decapentaplegic (Dpp) signaling and on the ventral side by epidermal growth factor receptor signaling (Goto and Hayashi 1997; Kubota et al. 2000), thus restricting the thoracic limb 
primordium to the ventral-lateral side. At embryonic stage 14 the cells of the wing primordium downregulate $\mathrm{Wg}$ signaling and separate from the leg primordium (Cohen et al. 1993). The cells of the leg primordium subdivide further into proximal and distal cells. $\mathrm{Wg}$ is also involved in this process by activating the late enhancer LT ("leg trigger") of $D l l$ in the distal cells, whereas the proximal cells do not require $w g$ function at this stage (McKay et al. 2009; Estella and Mann 2008; Estella et al. 2008). This function of $w g$ is separate from the early $w g$ function as evidenced by the fact that $\mathrm{Wg}$ cooperates with Dpp rather than being repressed by it (Held et al. 1994; Diaz-Benjumea et al. 1994; Penton and Hoffmann 1996; Jiang and Struhl 1996; Theisen et al. 1996; Lecuit and Cohen 1997; Theisen et al. 2007). Finally, $w g$ is then required for the specification of ventral fate in the legs (Struhl and Basler 1993; Theisen et al. 1996; Brook and Cohen 1996; Johnston and Schubiger 1996). This function, however, appears to be mechanistically different from the previous function where $\mathrm{Wg}$ protein forms a morphogen gradient. The ventral function of $w g$ is local and does not require $\mathrm{Wg}$ protein to form a morphogen gradient (DiazBenjumea and Cohen 1994; Wilder and Perrimon 1995; Theodosiou et al. 1998). Instead, Wg might interact with other local factors to specify ventral fate through its target gene $H 15$ (Wilder and Perrimon 1995). In summary, wg has three temporally separate and mechanistically different functions during the development of the Drosophila leg. The earliest function specifies and allocates the entire leg primordium including the proximal cells. By contrast, the later function in organizing pattern formation along the proximaldistal axis concerns only distal cells and the proximal cells do not require $w g$ at this stage. The ventral role, finally, is temporally and functionally separate from the proximaldistal role.

The $w g$ gene was the first segment polarity gene that has been shown to have a conserved expression pattern in longgerm and short-germ insects (Nagy and Carroll 1994). In the meantime, $w g$ has been isolated and studied in a number of other arthropods including different insect species (e.g. Jockusch et al. 2000; Niwa et al. 2000; Dearden and Akam 2001; Miyawaki et al. 2004), as well as myriapods (Hughes and Kaufman 2002; Prpic 2004; Janssen et al. 2004), crustaceans (Duman-Scheel et al. 2002; Nulsen and Nagy 1999; Prpic 2008), chelicerates (Damen 2002; Prpic et al. 2003), and an onychophoran (Eriksson et al. 2009). These data show that the expression pattern of $w g$ in all arthropods is highly conserved suggesting conserved functions. Surprisingly, however, functional tests have so far provided no support for highly conserved functions. Especially the three separate roles of $w g$ in leg development (allocation, distal, and ventral development) seem not to be conserved. In the milkweed bug Oncopeltus fasciatus and the cricket Gryllus bimaculatus wg is necessary for normal body segmentation, but does not seem to have a role in leg development (Angelini and Kaufman 2005a; Miyawaki et al. 2004). These two species belong to more basal hemimetabolous insect groups and Angelini and Kaufman (Angelini and Kaufman 2005b) have therefore suggested that the role of $w g$ in appendage development evolved only later in the holometabolous insects. Previous data from Tribolium castaneum indicated that in this holometabolous insect $w g$ is indeed required for appendage allocation, because legs were absent after $w g$ RNAi, but a role of $w g$ in distal and ventral development remained unclear (Ober and Jockusch 2006). We have therefore studied the temporal requirement of $w g$ in Tribolium leg development using staggered stage-specific RNAi. Our results show that in Tribolium $w g$ has three consecutive roles in allocation, distal, and ventral development, like in Drosophila. Our data suggest that the origin of these functions in insect evolution must predate the split between Diptera and Coleoptera.

\section{Materials and methods}

Parental and embryonic staggered RNAi

Parental RNAi was performed as described previously (Bucher et al. 2002). The pupae were injected with wg dsRNA with a concentration of $3,000 \mathrm{ng} / \mu \mathrm{l}$ and incubated at $32^{\circ} \mathrm{C}$ until eclosion. Eclosed females were mated with wild-type males and the eggs of two consecutive egg lays (24 h each) were collected. For embryonic RNAi, eggs were collected directly after egg deposition and incubated at $25^{\circ} \mathrm{C}$ until injection $(4,8,12$, and $18 \mathrm{~h}$ after egg laying). Injections were performed with beveled borosilicate needles using a micromanipulator and a FemtoJet injection controller (Eppendorf). Injected embryos were incubated at $25^{\circ} \mathrm{C}$ until hatching or (for in situ expression analysis) until germ band retraction. Removal of the wg mRNA below the level of detection was confirmed in each case by whole mount in situ hybridization using a $w g$ probe.

\section{Embryo fixation and in situ hybridization}

Embryos for in situ hybridization were dechorionized with a $50 \%$ solution of DanKlorix (Colgate-Palmolive) in water and fixed with $4 \%$ formaldehyde in a mixture of PEMS (0.1 M Pipes, $2 \mathrm{mM} \mathrm{MgSO} 4,1 \mathrm{mM}$ EDTA; $\mathrm{pH}=6.9$ ) and heptane. Vitelline membranes were removed by methanol shock and subsequent shearing through a syringe needle (19 G gauge). Whole-mount in situ hybridization detection of expression of enhanced green fluorescent protein (EGFP) mRNA was performed as described previously (Prpic et al. 2001). After in situ hybridization embryos were fixed with 
$4 \%$ formaldehyde in phosphate-buffered saline with $0.02 \%$ Tween-20, $\mathrm{pH}=7.4$ and embedded in $80 \%$ glycerol for microscopy.

\section{Microscopy and imaging}

Analysis of the cuticle markers (leg bristles) was performed with a laser scanning microscope (Zeiss LSM 510). Cuticles and embryos from the in situ hybridizations were observed with differential interference contrast microscopy (Zeiss Axioplan-2). Images were captured with an Intas digital camera and were subjected to adjustment of brightness, contrast, and color values using Adobe Photoshop image processing software (Version 7.0 for Apple Macintosh).

\section{Results}

A map of surface cuticle markers for the legs of $T$. castaneum

In order to identify leg parts in the appendages of Tribolium with morphological markers we obtained a comprehensive map of cuticle markers comprising sensorial bristles and campaniform sensillae (Fig. 1a, b). Each leg segment (podomere) has a characteristic set of cuticle markers. However, not all markers are present in all individuals or on all legs and sometimes the number of certain markers may differ between individuals.

The coxa has 10 cuticle markers, two of which are especially suitable, because they are present in all individuals (denoted by a red dot in Fig. 2). These two markers are bristles, cx-1 and cx-2, that are located close to each other on the anterior dorsal side of the coxa. Two groups of smaller bristles, cx-a and cx-p, are present in most individuals and mark the anterior and posterior part of the coxa, respectively. However, the number of bristles in each group is variable (see Fig. 2). The remaining bristles occur singly (except cx-bp which may be present as a pair) and are present in most individuals. The tiny ventral bristle cx-v, however, is more frequently missing on thoracic leg 1 than on legs 2 and 3 (boxed in Fig. 2). The trochanter has eight cuticle markers most of which are present in all individuals. The best trochanter marker is a group of campaniform sensillae, tr-cs, that may comprise two or three members (denoted by a red dot in Fig. 2). The dorsolateral bristle tr-l is less suited, because it is more frequently missing on thoracic legs 1 and 2 than on leg 3 (boxed in Fig. 2). The femur has 10 cuticle markers of which the long ventral bristle fe-v1 is the most conspicuous one and is present in all individuals. The
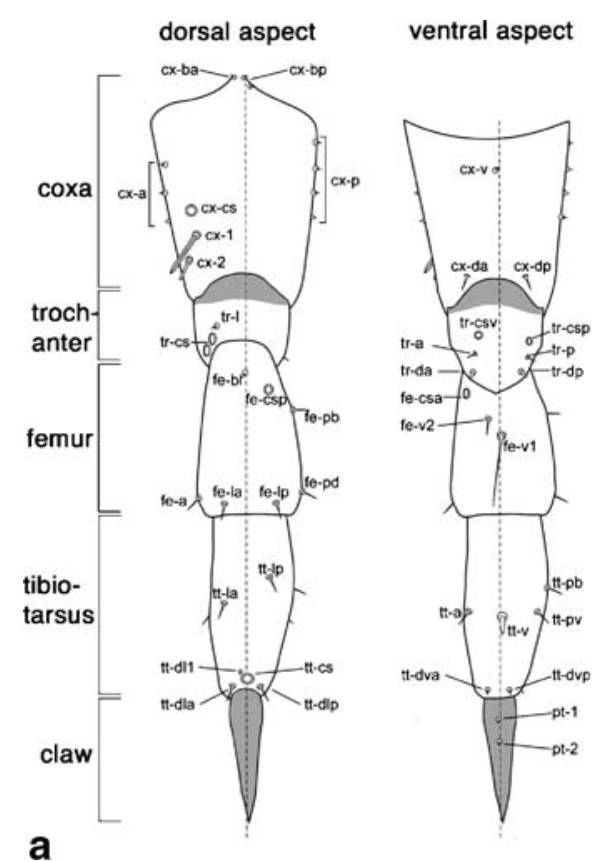

Fig. 1 A map of cuticular markers on the thoracic appendages of $T$ castaneum. a Schematic drawing of a leg in dorsal (left) and ventral (right) aspect. The podomeres are indicated on the left side. b Schematic map of a leg opened along the dorsal side. The ventral midline of the leg is indicated by the dotted line. The cuticular markers are named according to their position on a specific podomere. The letters before the dash stand for the podomere, the letters after the

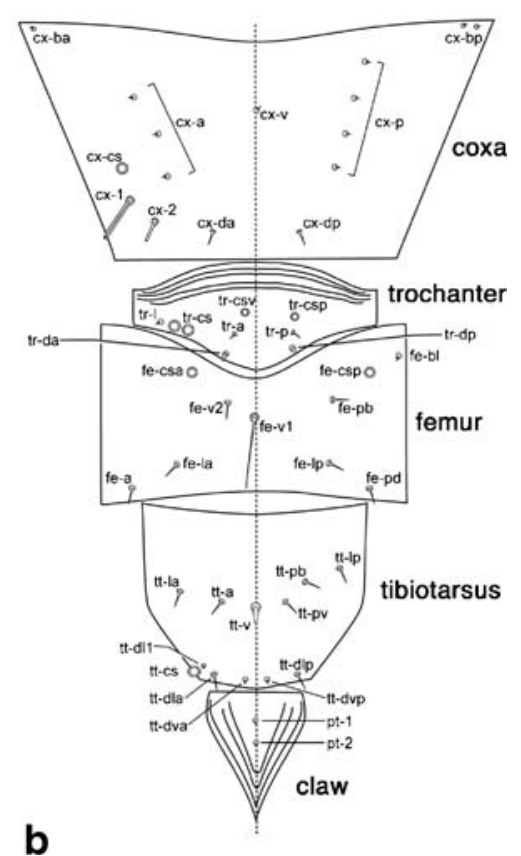

dash denote the position on that podomere. For example, fe-pd indicates that this marker is on the femur, and there it is located posterior and distal. Podomere abbreviations: $c x$ coxa, $t r$ trochanter, $f e$ femur, $t t$ tibiotarsus, $p t$ pretarsus (claw). Position abbreviations: $v$ ventral, $l$ dorsal, $a$ anterior, $p$ posterior, $b$ proximal, $d$ distal. Other abbreviations: cs campaniform sensilla 


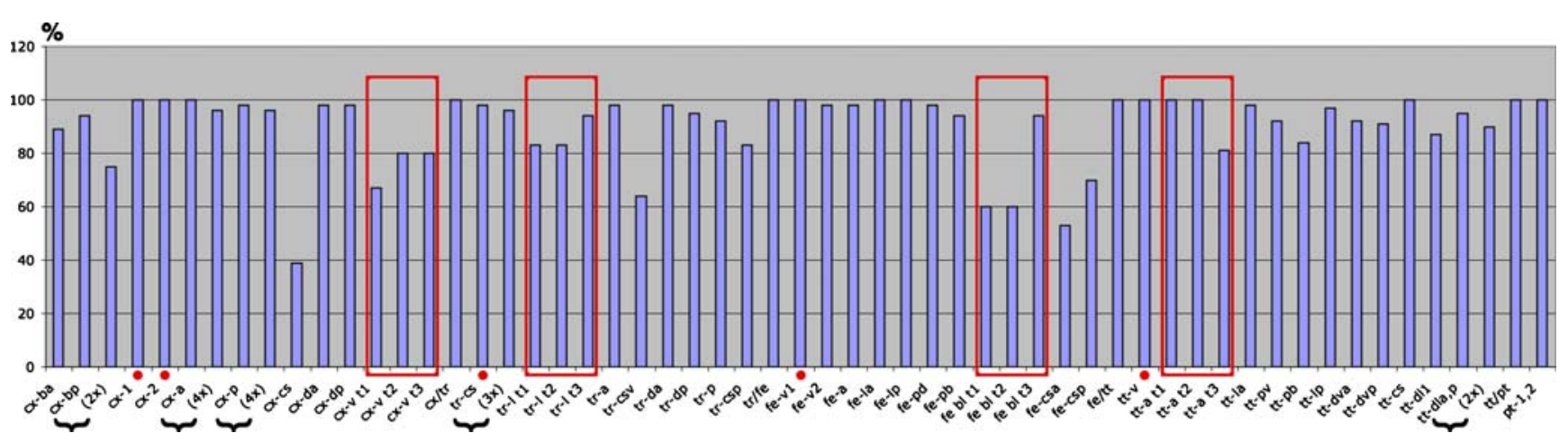

Fig. 2 Frequency of occurrence of cuticular leg markers on all three thoracic legs. The numbers were compiled from the study of 12 larvae. The cuticular markers are given on the $x$-axis (named according to the map in Fig. 1), and their frequency is given on the $y$-axis. Some markers may also be duplicated or quadruplicated; these cases are denoted by the bracket. A red box includes markers that have very different frequencies of occurrence on the different thoracic legs. The red dot denotes the most reliable and easy to discern markers for each podomere. Abbreviations: see Fig. 1

This indicates that $w g$ pRNAi has no effect on the eclosure rate or the subsequent survival of the imagines, but the injection per se leads to a slightly reduced rate of eclosure and survival. A significant effect was seen in the productivity of the females after injection. The uninjected control females layed approximately 13 eggs per female during the first egg-laying period after eclosure and this rate increased to approximately 17 eggs per female during the second egglaying period (Fig. 3b, left). In dsGFP-injected females the productivity was lower, probably owing to the injection procedure (Fig. 3b, center). However, productivity was even lower in females injected with $w g$ dsRNA (Fig. 3b, right). Thus, this effect cannot be attributed to injection stress alone, and must have been caused by the $w g$ dsRNA.

The layed eggs were incubated further and the rate of hatching was recorded. In the uninjected controls around half of the eggs were empty shells (hatching rate first egg lay, $61 \%$; second egg lay, $42 \%$; Fig. 3c). The percentage of empty egg shells increased in the GFP dsRNA-injected animals, and finally increased to over $95 \%$ in $w g$ dsRNA injected animals (Fig. 3c). All eggs that reached hatching in both controls and also in the experiment gave rise to wildtype larvae. These data indicate that pRNAi with $w g$ is leading to reduced female productivity (probably by interfering with gonad development or oogenesis), is embryonically lethal and the few obtained wild-type larvae in the $w g$ dsRNA-injected animals likely are escapers.

\section{Embryonic RNAi effects after staggered injections}

Since the pRNAi experiments lead to either empty egg shells or wild-type cuticles, we turned to staggered embryonic RNAi (eRNAi). eRNAi circumvents the problem of effects already during gonad formation or oogenesis, and by staggering the injections the effects that lead to early embryonic lethality can also be excluded. 

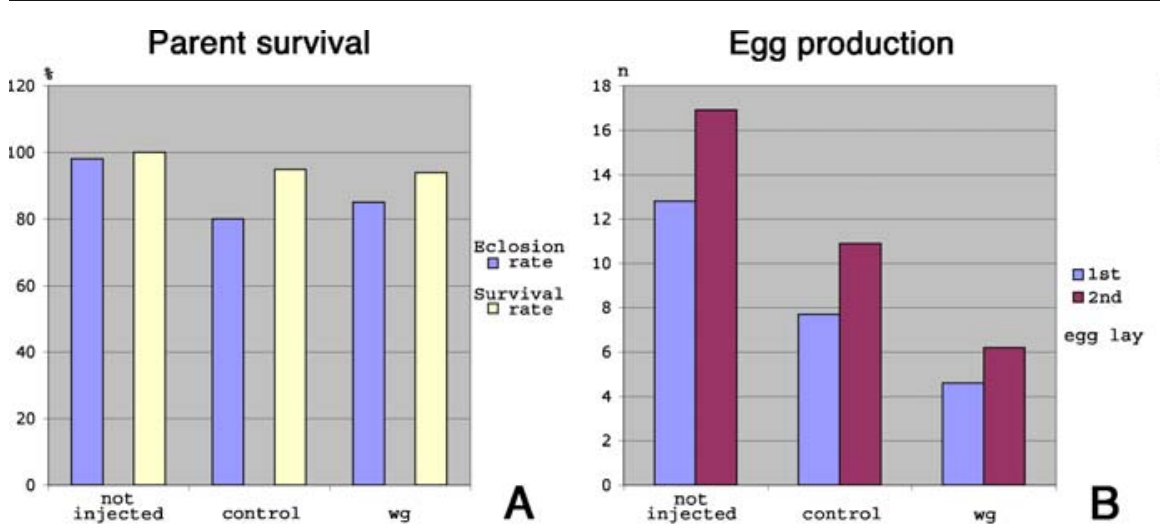

Fig. 3 Results of the parental RNAi experiments. a Eclosion rates and survival rates of the eclosed beetles (in percent). These are similar for not injected, control injected and $w g$ injected animals. b Female productivity measured after the first and second egg lay. The mean number of the eggs per female is given on the $y$-axis. $\mathbf{c}$ Hatching rate

We performed the earliest injections $4 \mathrm{~h}$ after egg laying. At this time point the embryo is still at a very early stage of development and the cleavage nuclei are in the process of approaching the egg periphery. Not unexpected therefore, these injections led to $90 \%$ empty egg shells (Fig. 4b) indicating early embryonic lethality similar to the results of the pRNAi. However, $10 \%$ of the larvae developed a cuticle. Three percent of these displayed only irregular cuticular structures (Fig. 5a) that were also present in the control injections (see Fig. 4a), 1\% were wildtype, and 6\% of the larvae had legs with severely disturbed proximaldistal axis formation (Fig. 5b, c). These results indicate that eRNAi can produce weaker phenotypes than pRNAi and that these weaker phenotypes include severe defects in morphology (including leg formation).

We have therefore performed staggered injections 8,12 , and $18 \mathrm{~h}$ after egg laying (these values are approximate values; actual injections may deviate from these values by $\pm 1.5 \mathrm{~h}$ ). At $8 \mathrm{~h}$ of development the embryo has reached the blastoderm stage, and we anticipated to circumvent in this way to interfere with very early $w g$ functions before blastoderm formation. At $12 \mathrm{~h}$ of development serosal closure takes place, the thoracic segments have already formed, but the leg primordia are not yet specified. We anticipated that in this way we could avoid to interfere with thoracic segment formation, but affect leg bud formation from the start. At $18 \mathrm{~h}$ of development germ band elongation is complete and all segments are formed, and the leg buds are already present. In this way we expected to be able to specifically interfere with late processes of leg development, without affecting segmentation or limb primordium specification. Injections later than $18 \mathrm{~h}$ after egg laying were not possible, because the vitelline membrane becomes too rigid and cannot be penetrated with the beveled borosilicate needles used in our experiments without squashing the (in percent) of the first and second egg lay and the percentage of wildtype cuticles after hatching. The hatching rate decreases dramatically after wg dsRNA injection, but all hatched larvae from uninjected, control injected and wg dsRNA injected mothers are wildtype. inj. injected

embryo. The results of the 8 -h injections were very similar to the 4 -h injections. There was no significant decrease of empty egg shells or increase of proximal-distal leg axis phenotypes (Figs. $4 \mathrm{~b}$ and $5 \mathrm{~d}-\mathrm{f}$ ). The 12-h injections lead to a strong decrease of the amount of empty egg shells, and the occurrence of proximo-distal leg phenotypes increased simultaneously to almost $30 \%$. This proximo-distal leg
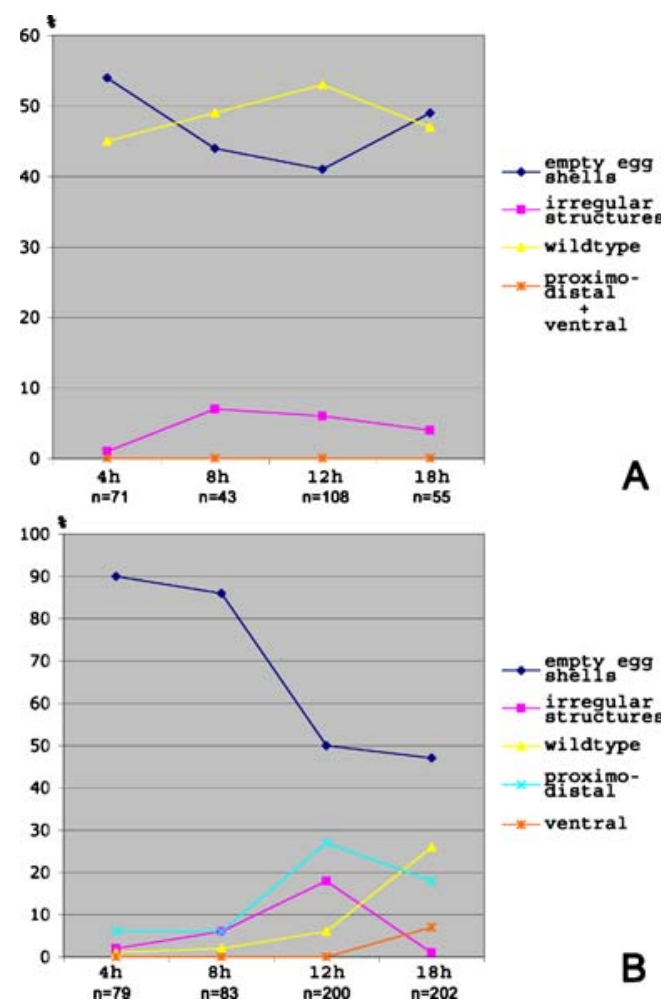

Fig. 4 Results of the staggered embryonic injections. a Control injections (injection buffer). b wg dsRNA injections. The time after egg laying is given on the $x$-axis. For details please refer to the text 

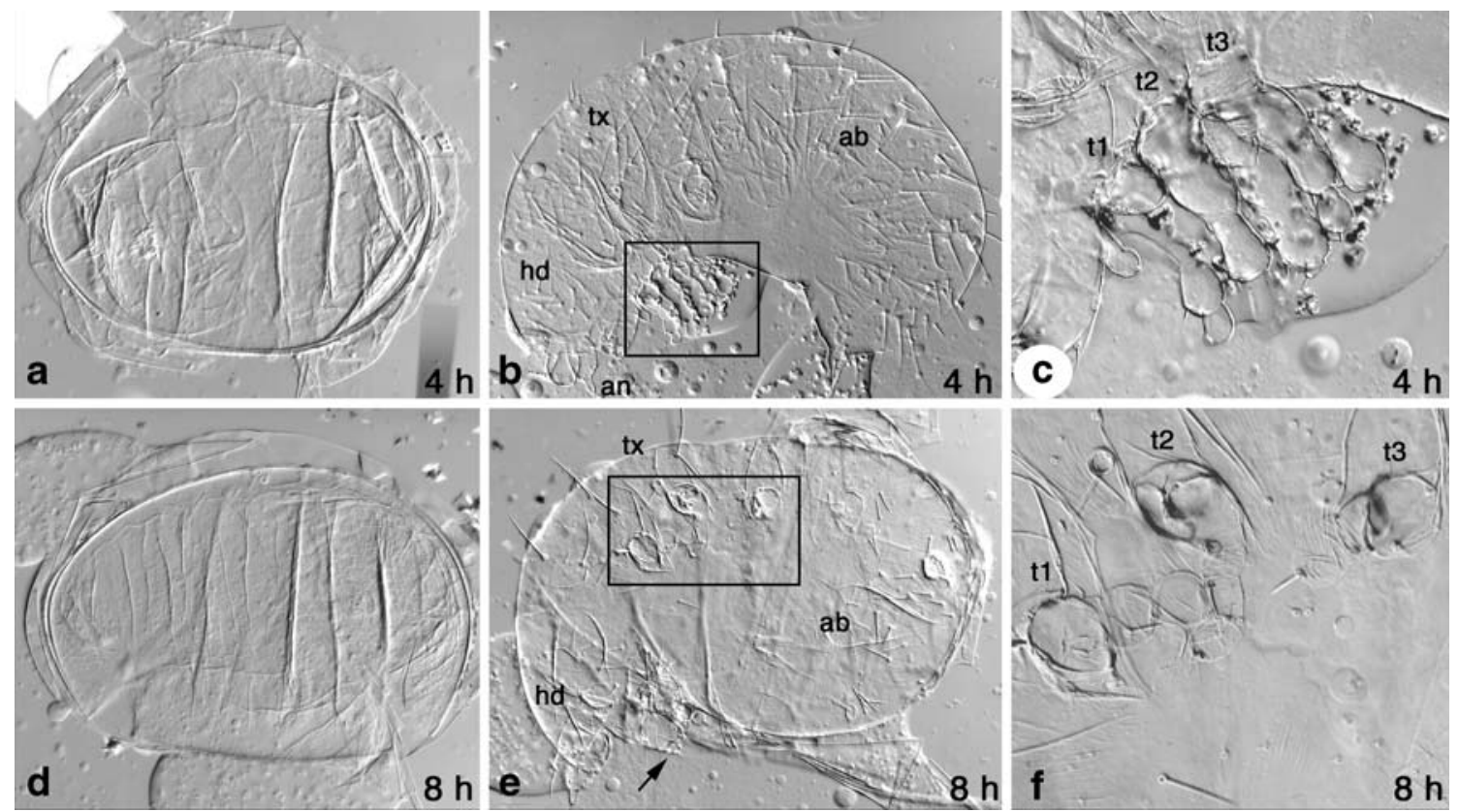

Fig. 5 Cuticle preparations of larvae hatched from eggs injected after $4(\mathbf{a}-\mathbf{c})$ and $8(\mathbf{d}-\mathbf{f})$ hours after egg laying. $\mathbf{a}$ and $\mathbf{d}$ are examples for eggs containing irregular cuticular structures. b and e show severely malformed larval cuticles with defective head, thorax, and abdomen. All appendages are affected; the thoracic legs are shown enlarged in panels (c) and (f). $\mathbf{c}$ is the enlargement of the area boxed in (b) and $\mathbf{f}$ is the enlargement of the area boxed in (e). The arrow in (e) points to the remnants of a maxilla. an antenna, $h d$ head, $t x$ thorax, $a b$ abdomen, $t 1$ prothoracic leg, $t 2$ mesothoracic leg, $t 3$ metathoracic leg phenotypes included specimens with malformed legs that nevertheless were composed of some leg segments (Fig. 6d, f), and specimens with more severely malformed legs (Fig. 6e, g). In these legs it was not possible to confidently establish the identity of the remaining leg portions because the morphology was too severely disturbed and most cuticle markers were absent. However, two additional phenotypes, that we term "candy cane" and "nonpareille" phenotypes, could be analyzed in more detail. In the "nonpareille" phenotype (named for Nonpareils pearls) all remaining leg segments appear rounded (Fig. 6b) and in severe cases they are lined up like pearls on a chain (Fig. 6c). In these phenotypes several cuticular markers were present (Fig. 6b, c) that indicate that the legs comprise a coxa with largely wild-type morphology, a long femur with ectopic constrictions, and a shortened tibiotarsus, and are lacking the claw. Thus, this phenotype reveals severe problems with proximal-distal axis formation distal to the coxa and distal-most structures are even lacking. The severe form of the "nonpareille" phenotype apparently is the "candy cane" phenotype where the podomeres do not form a chain anymore, but are fused into a long cane with a curiously bent distal end (Fig. 6a). Many of these specimens were simultaneously lacking the flagellum on the antennae (not shown).

In the 18-h injections the amount of empty egg shells dropped to control levels (Fig. 4a, b) and the amount of wildtype cuticles increased to almost $30 \%$, indicating that at these late stages many wg-dependent processes are already complete and cannot be disturbed by the injection of $w g$ dsRNA anymore. The overall amount of leg phenotypes remained constant, but the distal phenotype decreased and a novel, weaker phenotype appeared that does not lack distal leg segments. Instead, the claw is abnormally shaped: thin and pin-shaped and without the normal ventral bend (Fig. 7f). This phenotype also lacks the typical marker bristles that grow on, or very close to the ventral midline of the legs: on the femur (fe-v1) and tibiotarsus (tt-v, tt-pv). The lack of the ventral bend of the claw together with the lack of the ventral marker bristles indicates that this phenotype is caused by the loss of ventral tissue in the legs and we have therefore termed this phenotype "ventral-less".

The distal phenotypes of the 18-h injections were similar to the "nonpareille" phenotypes of the 12-h injections, but were weaker in most cases. The weakest cases had all typical podomeres including the claw, but the femur was elongated and thinner than in the wildtype and the tibiotarsus showed ectopic constrictions (Fig. 7d). The intermediate phenotype had normal coxa and trochanter, but a shortened tibiotarsus, no claw and a single ectopic constriction in the femur (Fig. 7b), and the most severe phenotypes were "nonpareille" phenotypes with pearlshaped tibiotarsus and rounded ectopic subdivisions of the femur, and the trochanter seems to be fused entirely to the coxa (Fig. 7c). 
Fig. 6 Thoracic appendages of larvae hatched from eggs injected after $12 \mathrm{~h}$ after egg laying. a Typical "candy cane" leg phenotype. b, c "Nonpareille" leg phenotypes. Note the additional constrictions in the femur and the absence of the pretarsal claw. d-g More severe truncations of the legs. In some cases only stumps of the legs remain $(\mathbf{e}, \mathbf{g})$, in other cases some leg segments remain, but cannot be identified because of the lack of cuticle markers $(\mathbf{d}, \mathbf{f})$. For the labeling of the cuticle markers please refer to Fig. 1. fe femur, $t t$ tibiotarsus
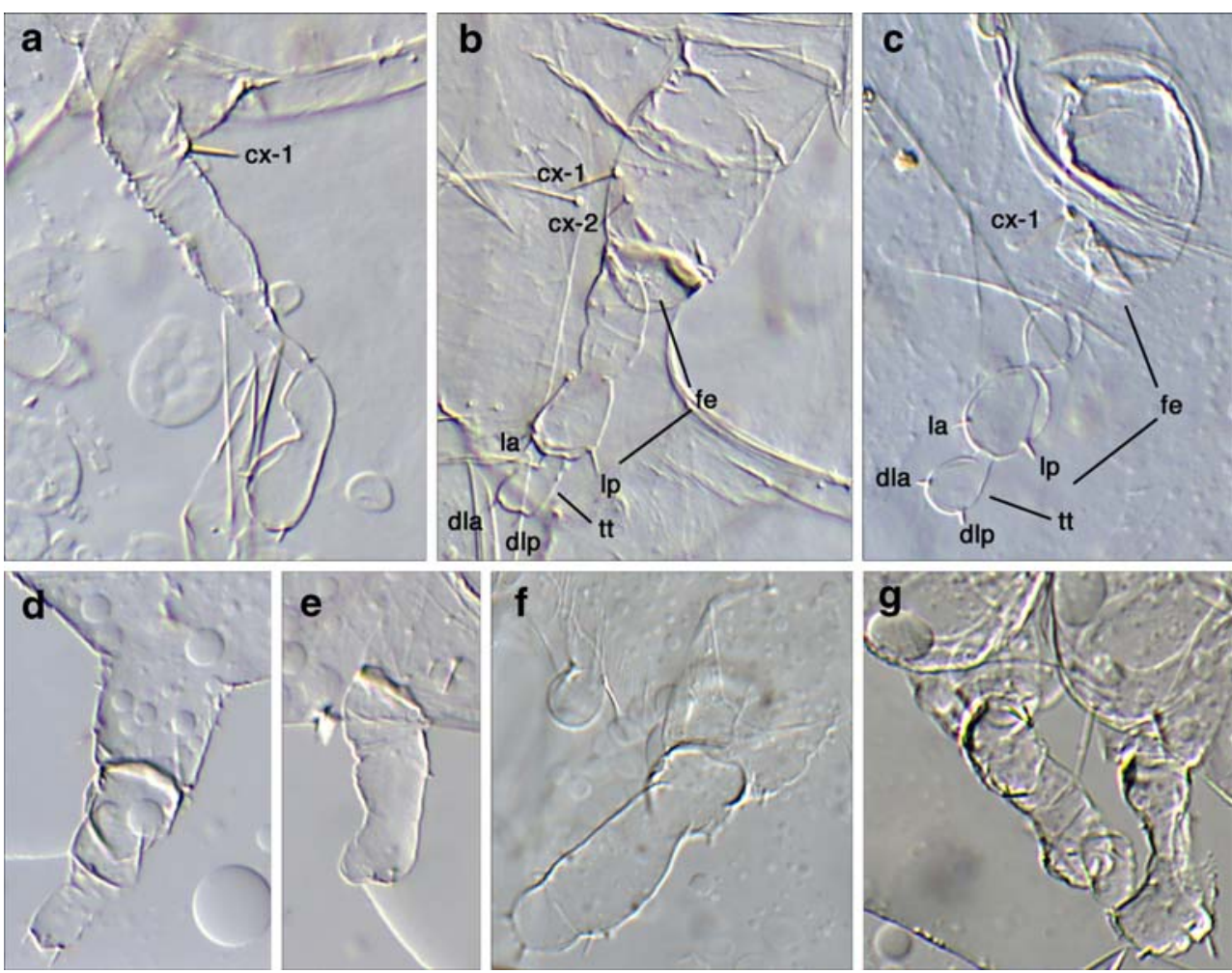

We have then used molecular markers for the ventral and distal tissues in the developing Tribolium leg. The GEKU insertional mutagenesis screen has produced about 50 enhancer trap lines with EGFP expression in the legs (Trauner et al. 2009). We have used two of these lines to perform staggered RNAi with wg. The line Goe-04609 expresses EGFP mRNA along the ventral side of the legs, and in addition in a segmentally repeated pattern and in the developing heart (Fig. 8a). In embryos injected $18 \mathrm{~h}$ after egg laying the expression is largely identical to the expression in the wildtype, but the ventral expression in the legs is lacking (Fig. 8b). This demonstrates that ventral leg tissue is missing. In the embryos injected earlier, the pattern of Goe-04609 is disturbed more severely: the segmental pattern is fused along the anterior-posterior axis and the expression in the heart is missing (Fig. 8c). The line Goe-12407 expresses EGFP mRNA in a ring of cells near the tips of the legs, and in addition in a punctate pattern in the central nervous system (Fig. 8d). In embryos injected $18 \mathrm{~h}$ after egg laying the expression is very similar to the expression in the wildtype, but the distal ring in the legs is reduced to a spot of expression on the dorsal side of the legs (Fig. 8e). This indicates that ventral leg tissue is missing. In the embryos injected earlier, the pattern of Goe12407 is weak (Fig. 8f). However, the expression in the antennae is much stronger, indicating that the enhancer trapped in Goe-12407 is normally repressed in the antenna by $w g$.

\section{Discussion}

Evidence for a role of $w g$ in leg allocation in Tribolium

Our experiments with pupal injections were not able to reveal a function of $w g$ in leg development. Despite good eclosion and survival rates, the females that eclosed from pupae injected with $w g$ dsRNA had a very low productivity and from the few eggs almost no larvae hatched; those larvae that did hatch were wildtype. The low productivity suggests that $w g$ might have a role already during oogenesis. The low hatching rate indicates that $w g$ has functions early in development that lead to early embryonic lethality and the few wild-type larvae must be escapers that by chance did not receive $w g$ dsRNA.

Early injections into eggs lead to similar effects. Most of the eggs of the 4- and 8-h injections were empty or contained irregular cuticular structures similar to those observed by Bolognesi et al. (2008). The few larvae that developed showed severe phenotypes indicating problems with several developmental processes including segmentation. The legs of these larvae were severely malformed. The defects we have observed are very similar to those reported by Ober and Jockusch (2006). These authors also found segmentation defects and severely truncated or missing legs after $w g$ RNAi. The specimens figured in their Fig. $2 \mathrm{~d}-\mathrm{g}$ are very similar to our results after early injections after 4 and $8 \mathrm{~h}$. The specimen figured in their Fig. $2 \mathrm{i}$ shows 
Fig. 7 Thoracic appendages of larvae hatched from eggs injected after $18 \mathrm{~h}$ after egg laying. a Wild-type larval leg for comparison. b-d Different "nonpareille" phenotypes. Note the additional constrictions in the femur and the absence of the pretarsal claw in the stronger "nonpareille" phenotype $(\mathbf{b}, \mathbf{c})$ and the additional constrictions in the tibiotarsus and the presence of the pretarsal claw in the weaker "nonpareille" phenotype (d). Also note the presence of a largely normal coxa in these phenotypes. (e, $\left.E^{\prime}\right)$ Wild-type larval leg for comparison and labeled for the podomeres (e) and the cuticle markers of the femur and tibiotarsus $\left(E^{\prime}\right)$. f A "ventral-less" phenotype labeled for the cuticle markers of the femur and the tibiotarsus. Note the absence of fe-v1, tt-pv, and $\mathrm{tt}-\mathrm{v}$. Also note the lacking ventral bend of the pretarsal claw. For the labeling of the cuticle markers please refer to Fig. 1. $c x$ coxa, $t r$ trochanter, $f e$ femur, $t t$ tibiotarsus, $\mathrm{cl}$ pretarsal claw
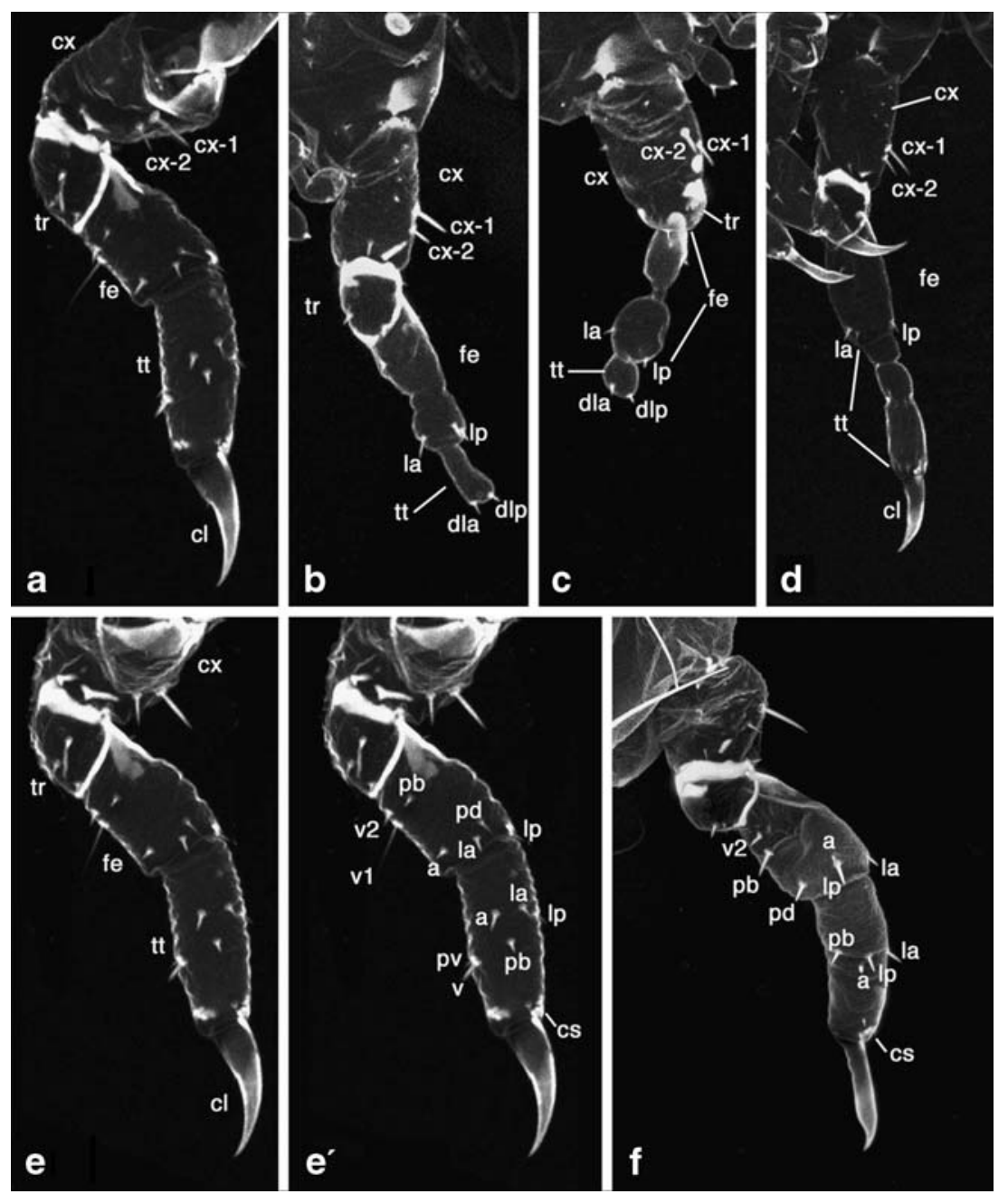

shortened and severely malformed appendages, which is also very similar to what we have found in the early injections. Ober and Jockusch (2006) have interpreted these phenotypes as support for a role of $w g$ in appendage allocation. However, because in all of these specimens the segments are also malformed, it is unclear whether the leg defects are true leg developmental defects or secondary defects because of the defective segment formation.

We avoided interfering with the early functions of $w g$ in body segmentation by applying later injections after 12 and $18 \mathrm{~h}$. The most severe leg phenotypes in these injections are seen in the 12-h injections: the legs are malformed and all parts of the leg are affected including the coxa. This is very similar to the early function of $w g$ in leg allocation in Drosophila when Wg is required for activating the 304 enhancer of $D l l$ in all cells of the leg primordium including proximal (coxal) cells (Cohen et al. 1993; McKay et al. 2009). We thus interpret the severe malformations in 12-h injections as evidence for a role of Tribolium $w g$ in the specification and allocation of the entire leg primordium.
Separate functions of $w g$ in distal and ventral development

In the "candy cane" and "nonpareille" phenotypes in the 12and 18-h injections the coxa is not affected indicating that at this stage of development $w g$ is not required for the development of the proximal leg parts anymore. The distal leg parts, however, are malformed and the distal-most portion, the claw, is missing in most specimens. This distal function is very similar to the distal function in Drosophila leg imaginal precursors where $w g$ is required for the development of all leg portions distal to the coxa by activating the LT enhancer of Dll (Cohen et al. 1993; Estella et al. 2008; McKay et al. 2009). The extra constrictions in the femur or the tibiotarsus in the strong and weak "nonpareille" phenotypes, respectively, are unexpected and their origin is unclear. The normal number of constrictions (future joints) in Drosophila is specified by the action of the Notch pathway (de Celis et al. 1998; Rauskolb and Irvine 1999; Bishop et al. 1999) which is regulated by the leg gap genes and these are regulated by $\mathrm{Wg}$ signaling in conjunction with Dpp signaling (Lecuit and Cohen 1997; Estella and Mann 2008). It is 
Fig. 8 Expression of molecular markers for ventral and distal leg fate is altered in $w g$ RNAi embryos. a-c Expression of the ventral leg marker enhancer trap Goe-04609 in wild-type embryos (a) and embryos injected after 18 (b) and $12 \mathrm{~h}$ (c) after egg laying. d-f Expression of the distal leg marker enhancer trap Goe-12407 in wild-type embryos (d) and embryos injected after 18 (e) and $12 \mathrm{~h}(\mathbf{f})$ after egg laying. $t 1$ prothoracic leg, $t 2$ mesothoracic leg, $t 3$ metathoracic leg. Anterior is to the left in all panels
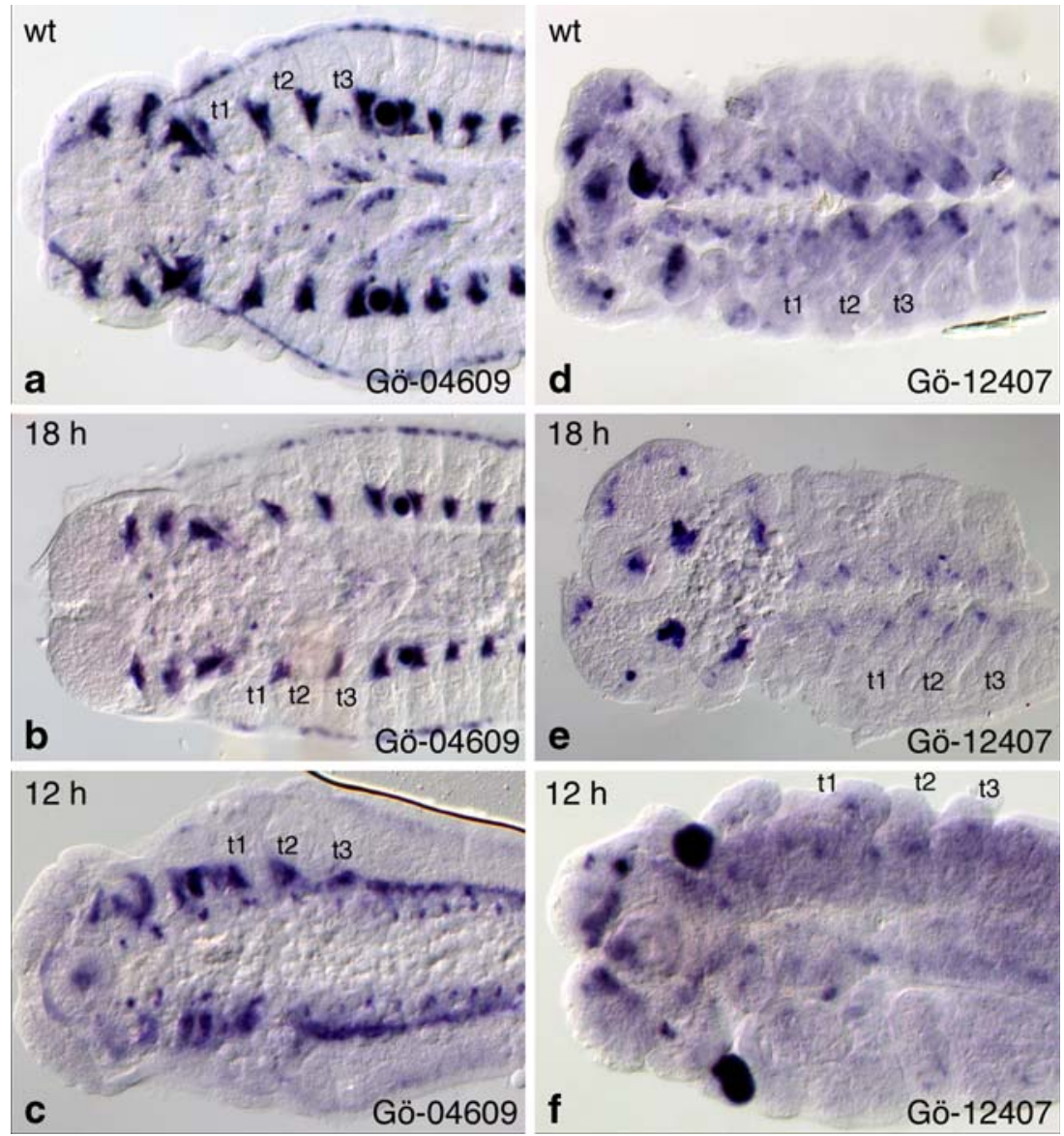

currently unknown whether a similar cascade exists in Tribolium, but this is very likely because the role of the Notch pathway in leg segmentation is also conserved in the spider Cupiennius salei, a basally branching arthropod (Prpic and Damen 2009). Thus, it is possible that interference with the distal function of $w g$ disturbs the normal segmentation of the Tribolium legs leading to ectopic constrictions in the distal segments.

The "ventral-less" phenotype appeared only in the 18-h injections. In these legs all segments are normal except that the ventral-most tissue is lacking. This ventral function appears to be separate from the distal function since the "ventral-less" phenotype does not show any distal defects. Again this is very similar to the ventral function of $w g$ in Drosophila where Wg does not act as a concentration-dependent morphogen, but rather acts locally on the ventral side probably in conjunction with other ventrally expressed factors (Diaz-Benjumea and Cohen 1994; Wilder and Perrimon 1995; Theodosiou et al. 1998).

\section{Conclusions}

In summary, our results indicate three separate functions of $w g$ in leg development of Tribolium, and these functions also are temporally separated. The early function of $w g$ is the specification of the entire leg primordium. This is supported by the strong leg defects after the 12-h injections that affect all leg parts including the coxa. Later injections cannot interfere with normal coxa development (the "candy cane" and "nonpareille" phenotypes in which distal leg portions are affected, but the coxa is normal) suggesting that at this stage $w g$ has a role in the formation of the entire distal leg portion, but not in the proximal cells anymore. Future studies have to reveal whether this distal function is performed by $w g$ as a morphogen in conjunction with $d p p$ as the second morphogen as it is the case in Drosophila (e.g. Lecuit and Cohen 1997). Finally, the late function of $w g$ is the specification of ventral leg cells. This function is independent of the distal function, because in the "ventral-less" phenotype the formation of the proximal-distal axis is not affected. The fact that the effect is restricted to ventral leg cells is evidence that it does not involve a $\mathrm{Wg}$ gradient.

Our results indicate that the separate functions of $w g$ in leg allocation, distal, and ventral leg development must have evolved before the split between Diptera and Coleoptera. Without further information from more basal insects or non-insect arthropods this suggests that the leg functions of $w g$ have evolved in the last common ancestor 
of the holometabolous insects and thus earlier than suggested by Angelini and Kaufman (2005b). In order to exactly pinpoint the origin of the involvement of $w g$ in arthropod leg development, the function of $w g$ has to be studied in additional holo- and hemimetabolous insect species and also in other arthropod groups where functional data are currently lacking.

Acknowledgements We thank Gregor Bucher, Ernst A. Wimmer, and Johannes Schinko for providing us with the enhancer trap lines Goe-04609 and Goe-12407 from the GEKU insertional mutagenesis screen. We especially thank Martin Klingler for his support and advice. We also thank the members of our group for stimulating discussions and Marco Winkler for technical assistance. This work has been supported by the Deutsche Forschungsgemeinschaft (grant number PR 1109/1-1).

Open Access This article is distributed under the terms of the Creative Commons Attribution Noncommercial License which permits any noncommercial use, distribution, and reproduction in any medium, provided the original author(s) and source are credited.

\section{References}

Angelini DR, Kaufman TC (2005a) Functional analyses in the milkweed bug Oncopeltus fasciatus (Hemiptera) support a role for Wnt signaling in body segmentation but not appendage development. Dev Biol 283:409-423

Angelini DR, Kaufman TC (2005b) Insect appendages and comparative ontogenetics. Dev Biol 286:57-77

Bishop SA, Klein T, Martinez Arias A, Couso JP (1999) Composite signalling from Serrate and Delta establishes leg segments in Drosophila through Notch. Development 126:2993-3003

Bolognesi R, Farzana L, Fischer TD, Brown SJ (2008) Multiple Wnt are required for segmentation in the short-germ embryo of Tribolium castaneum. Curr Biol 18:1624-1629

Brook WJ, Cohen SM (1996) Antagonistic interactions between Wingless and Decapentaplegic responsible for dorsal-ventral pattern in the Drosophila leg. Science 273:1373-1377

Bucher G, Scholten J, Klingler M (2002) Parental RNAi in Tribolium (Coleoptera). Curr Biol 12:R85-R86

Campbell G, Weaver T, Tomlinson A (1993) Axis specification in the developing Drosophila appendages: the role of wingless, decapentaplegic, and the homeobox gene aristaless. Cell 74:1113-1123

Cohen B, Simcox AA, Cohen SM (1993) Allocation of the thoracic imaginal primordia in the Drosophila embryo. Development 117:597-608

Damen WGM (2002) Parasegmental organization of the spider embryo implies that the parasegment is an evolutionary conserved entity in arthropod embryogenesis. Development 129:1239-1250

de Celis JF, Tyler DM, de Celis J, Bray SJ (1998) Notch signalling mediates segmentation of the Drosophila leg. Development 125:4617-4626

Dearden PK, Akam M (2001) Early patterning in the grasshopper, Schistocerca gregaria: wingless, decapentaplegic and caudal expression. Development 128:3435-3444

Diaz-Benjumea FJ, Cohen SM (1994) wingless acts through the shaggy/zeste-white 3 kinase to direct dorsal-ventral axis formation in the Drosophila leg. Development 120:1661-1670

Diaz-Benjumea FJ, Cohen B, Cohen SM (1994) Cell interactions between compartments establishes the proximal-distal axis of Drosophila legs. Nature 371:175-179
Duman-Scheel M, Pirkl N, Patel NH (2002) Analysis of the expression pattern of Mysidium columbiae wingless provides evidence for conserved mesodermal and retinal patterning processes among insects and crustaceans. Dev Genes Evol 212:114-123

Eriksson J, Tait NN, Budd GE, Akam M (2009) The involvement of engrailed and wingless during segmentation in the onychophoran Euperipatoides kanangrensis (Peripatopsidae: Onychophora) (Reid, 1996). Dev Genes Evol 219:249-264

Estella C, Mann RS (2008) Logic of Wg and Dpp induction of distal and medial fates in the Drosophila leg. Development 135:627-636

Estella C, McKay DJ, Mann RS (2008) Molecular integration of Wingless, Decapentaplegic, and autoregulatory inputs into Distalless during Drosophila leg development. Dev Cell 14:86-96

Goto S, Hayashi S (1997) Specification of the embryonic limb primordium by graded activity of Decapentaplegic. Development 124:125-132

Held LI, Heup MA, Sappington M, Peters SD (1994) Interactions of decapentaplegic, wingless, and Distal-less in the Drosophila leg. Roux Arch Dev Biol 203:310-319

Hughes CL, Kaufman TC (2002) Exploring myriapod segmentation: the expression patterns of even-skipped, engrailed, and wingless in a centipede. Dev Biol 247:47-61

Janssen R, Prpic NM, Damen WGM (2004) Gene expression suggests decoupled dorsal and ventral segmentation in the millipede Glomeris marginata (Myriapoda: Diplopoda). Dev Biol 268:89-104

Jiang J, Struhl G (1996) Complementary and mutually exclusive activities of Decapentaplegic and Wingless organize axial patterning during Drosophila leg development. Cell 86:401-409

Jockusch EL, Nulsen C, Newfeld SJ, Nagy LM (2000) Leg development in flies versus grasshoppers: differences in $d p p$ expression do not lead to differences in the expression of downstream components of the leg patterning pathway. Development 127:1617-1626

Johnston LA, Schubiger G (1996) Ectopic expression of wingless in imaginal discs interferes with decapentaplegic expression and alters cell determination. Development 122:3519-3529

Kubota K, Goto S, Eto K, Hayashi S (2000) EGF receptor attenuates Dpp signaling and helps to distinguish the wing and leg cell fates in Drosophila. Development 127:3769-3776

Kubota K, Goto S, Hayashi S (2003) The role of Wg signaling in the patterning of embryonic leg primordium in Drosophila. Dev Biol 257:117-126

Lecuit T, Cohen SM (1997) Proximal-distal axis formation in the Drosophila leg. Nature 388:139-145

McKay DJ, Estella C, Mann RS (2009) The origins of the Drosophila leg revealed by the cis-regulatory architecture of the Distalless gene. Development 136:61-71

Miyawaki K, Mito T, Sarashina I, Zhang H, Shinmyo Y, Ohuchi H, Noji S (2004) Involvement of Wingless/Armadillo signaling in the posterior sequential segmentation in the cricket, Gryllus bimaculatus (Orthoptera), as revealed by RNAi analysis. Mech Dev 121:119-130

Nagy LM, Carroll SB (1994) Conservation of wingless patterning functions in the short-germ embryos of Tribolium castaneum. Nature 367:460-463

Niwa N, Inoue $\mathrm{Y}$, Nozawa A, Saito M, Misumi Y, Ohuchi H, Yoshioka H, Noji S (2000) Correlation of diversity of leg morphology in Gryllus bimaculatus (cricket) with divergence in $d p p$ expression pattern during leg development. Development 127:4373-4381

Nulsen C, Nagy LM (1999) The role of wingless in the development of multibranched crustacean limbs. Dev Genes Evol 209:340-348

Ober KA, Jockusch EL (2006) The roles of wingless and decapentaplegic in axis and appendage development in the red flour beetle, Tribolium castaneum. Dev Biol 294:391-405 
Penton A, Hoffmann FM (1996) Decapentaplegic restricts the domain of wingless during Drosophila limb patterning. Nature 382:162-165

Prpic NM (2004) Homologs of wingless and decapentaplegic display a complex and dynamic expression profile during appendage development in the millipede Glomeris marginata (Myriapoda: Diplopoda). Front Zool 1:6. doi:10.1186/1742-9994-1-6

Prpic NM (2008) Parasegmental appendage allocation in annelids and arthropods and the homology of parapodia and arthropodia. Front Zool 5:17. doi:10.1186/1742-9994-5-17

Prpic NM, Damen WGM (2009) Notch-mediated segmentation of the appendages is a molecular phylotypic trait of the arthropods. Dev Biol 326:262-271

Prpic NM, Wigand B, Damen WGM, Klingler M (2001) Expression of dachshund in wild-type and Distal-less mutant Tribolium corroborates serial homologies in insect appendages. Dev Genes Evol 211:467-477

Prpic NM, Janssen R, Wigand B, Klingler M, Damen WGM (2003) Gene expression in spider appendages reveals reversal of exd/hth spatial specificity, altered leg gap gene dynamics, and suggests divergent distal morphogen signaling. Dev Biol 264:119-140

Rauskolb C, Irvine KD (1999) Notch-mediated segmentation and growth control of the Drosophila leg. Dev Biol 210:339-350
Sanson B (2001) Generating patterns from field of cells. Examples from Drosophila segmentation. EMBO rep 2:1083-1088

Struhl G, Basler K (1993) Organizing activity of Wingless protein in Drosophila. Cell 72:527-540

Theisen H, Haerry TE, O'Connor MB, Marsh JL (1996) Developmental territories created by mutual antagonism between Wingless and Decapentaplegic. Development 122:3939-3948

Theisen H, Syed A, Nguyen BT, Lukacsovich T, Purcell J, Srivastava GP, Iron D, Gaudenz K, Nie Q, Wan FYM, Waterman ML, Marsh JL (2007) Wingless directly represses DPP morphogen expression via an Armadillo/TCF/Brinker complex. PLoS ONE 2:e142

Theodosiou NA, Zhang S, Wang WY, Xu T (1998) slimb coordinates wg and $d p p$ expression in the dorsal-ventral and anterior-posterior axes during limb development. Development 125:3411-3416

Trauner J, Schinko J, Lorenzen MD, Shippy TD, Wimmer EA, Beeman RW, Klingler M, Bucher G, Brown SJ (2009) Largescale insertional mutagenesis of a coleopteran stored grain pest, the red flour beetle Tribolium castaneum, identifies embryonic lethal mutations and enhancer traps. BMC Biol 7:73

Wilder EL, Perrimon N (1995) Dual functions of wingless in the Drosophila leg imaginal disc. Development 121:477-488 